\title{
CONSTRUCTING POLYNOMIAL SPECTRAL MODELS FOR STARS
}

\author{
Hans-Walter Rix ${ }_{1}^{1}$, Yuan-Sen Ting (丁源森) ${ }^{2}$, Charlie Conroy ${ }^{2}$, and David W. Hogg ${ }^{1,3,4,5}$ \\ ${ }^{1}$ Max Planck Institute for Astronomy, Königstuhl 17, D-69117 Heidelberg, Germany \\ ${ }^{2}$ Harvard-Smithsonian Center for Astrophysics, 60 Garden Street, Cambridge, MA 02138, USA \\ ${ }^{3}$ Simons Center for Data Analysis, 160 Fifth Avenue, 7th floor, New York, NY 10010, USA \\ ${ }^{4}$ Center for Cosmology and Particle Physics, Department of Physics, New York University, 4 Washington Place, Room 424, New York, NY 10003, USA \\ ${ }^{5}$ Center for Data Science, New York University, 726 Broadway, 7th floor, New York, NY 10003, USA \\ Received 2016 March 21; revised 2016 May 9; accepted 2016 May 9; published 2016 July 26
}

\begin{abstract}
Stellar spectra depend on the stellar parameters and on dozens of photospheric elemental abundances. Simultaneous fitting of these $\mathcal{N} \sim 10-40$ model labels to observed spectra has been deemed unfeasible because the number of ab initio spectral model grid calculations scales exponentially with $\mathcal{N}$. We suggest instead the construction of a polynomial spectral model (PSM) of order $\mathcal{O}$ for the model flux at each wavelength. Building this approximation requires a minimum of only $\left(\begin{array}{c}\mathcal{N}+\mathcal{O} \\ \mathcal{O}\end{array}\right)$ calculations: e.g., a quadratic spectral model $(\mathcal{O}=2)$ to fit $\mathcal{N}=20$ labels simultaneously can be constructed from as few as 231 ab initio spectral model calculations; in practice, a somewhat larger number $(\sim 300-1000)$ of randomly chosen models lead to a better performing PSM. Such a PSM can be a good approximation only over a portion of label space, which will vary case-by-case. Yet, taking the APOGEE survey as an example, a single quadratic PSM provides a remarkably good approximation to the exact ab initio spectral models across much of this survey: for random labels within that survey the PSM approximates the flux to within $10^{-3}$ and recovers the abundances to within $\sim 0.02$ dex rms of the exact models. This enormous speed-up enables the simultaneous many-label fitting of spectra with computationally expensive ab initio models for stellar spectra, such as non-LTE models. A PSM also enables the simultaneous fitting of observational parameters, such as the spectrum's continuum or line-spread function.
\end{abstract}

Key words: methods: data analysis - stars: abundances - stars: atmospheres - techniques: spectroscopic

\section{FITTING STELLAR SPECTRA}

The spectra of stars encode an enormous amount of information, mainly about the stars' current physical state and the composition of the chemical elements in their photosphere. But the number of stellar labels ${ }^{6}$ that fully specify a spectrum is large: a handful of stellar parameters and much of the periodic table. We know that stellar spectra with $\mathrm{S} / \mathrm{N} \sim 100$ and $R \sim 20,000-40,000$, currently emerging for $10^{4-6}$ objects from various surveys, contain the information to constrain 10-40 labels, at least for stars with favorable effective temperatures, 4000-7000 K (e.g., Smiljanic et al. 2014; García Pérez et al. 2016; Sheinis et al. 2015). The accuracy and precision of label estimates for vast stellar samples matters greatly for understanding the formation of the Galaxy, stellar physics, and the origin of the chemical elements (e.g., Freeman \& Bland-Hawthorn 2002; Rix \& Bovy 2013; Frebel \& Norris 2015).

A principled determination of these stellar labels requires fitting the data with physical model spectra, in which the stellar labels constitute 10-40 model parameters. The calculation of such ab initio spectral models (a.i. models) through radiative transfer calculations has a storied tradition (for an overview, see Smiljanic et al. 2014; García Pérez et al. 2016). Current a.i. models vary by the degree of physical simplification they apply: LTE versus non-LTE; plane-parallel versus spherical geometry; 1D, averaged or full 3D; static versus time dependent; and by the extent and robustness of the atomic data that underlie them.

\footnotetext{
6 We use the term "labels" to mean the union of stellar parameters and photospheric elemental abundances because in the current context these two classes of stellar attributes are being treated equivalently.
}

The computation of a.i. models is expensive, all the more so if the simplifying assumptions are dropped. This is why "brute force" fitting of spectra with a.i. models (of, say, 10-40 labels) is unfeasible for the foreseeable future: most approaches to fitting a.i. models to observed spectra have relied on precomputing grids of a.i. spectra in the $\mathcal{N}$-dimensional label space, and then interpolating between them pixel-by-pixel, e.g., quadratically (i.e., second-order) or cubic (i.e., third-order) as in Allende Prieto et al. (2006, 2014). However, for any number of grid points, $M \approx 3-5$, in each label dimension, the total number of a.i. model calculations required grows exponentially with the dimension $\mathcal{N}$ of label space: $N_{\text {tot }} \propto M^{\mathcal{N}} \propto \exp (\mathcal{N} \cdot \ln M)$. Established approaches have coped with this in practice by fitting model spectra first in a three- to six-dimensional sub-space of $\mathcal{N}$, and subsequently fitting one (or two) further labels at a time, holding the initial labels fixed. This approach has important limitations with with state-of-the-art data: first, Ting et al. (2016, hereafter T16) have shown that more than just two or three elemental abundances affect the atmosphere structure, and hence are physically covariant with the basic stellar parameters; second, physical correlations and data-driven covariances are known to exist among (abundance) labels, but cannot be estimated when fitting one label at a time; third, to mitigate against unaccounted covariances, established fitting approaches have often focused on unblended lines, thereby underexploiting the information content of the data by a large factor (T16).

T16 proposed a way to overcome this impasse by employing more linear algebra in the fitting to save on a.i. model calculations; in this Letter, we take this idea a step further. T16 proposed tessellating the space of stellar labels into a finite set of regions (dubbed linear Taylor-spheres, or 1OTS). Within 
each 1OTS, the a.i. model flux at each wavelength can be described sufficiently well by a linearized spectral model (LSM), linearized (in all labels) around the a.i. model spectrum at a fiducial label value (see also Recio-Blanco et al. 2006). T16 showed that such LSM can sufficiently approximate the exact model spectra within a 1OTS. Together with the finite number of Taylor-spheres required to cover any given spectral survey (e.g., 150 for the APOGEE red clumps), this leads to a dramatic reduction in the total number of a.i. model calculations: simultaneous fitting of 10-40 labels should then be feasible.

Here, we point out a rather obvious extension of this idea, which yields even greater computational savings: the construction of approximate model spectra, where the predicted flux at each pixel by a polynomial in all labels away from a fiducial model spectrum. This idea had been put forth by Prugniel et al. (2011) for empirical spectra, who did not, however, pursue its potential of fitting many labels simultaneously. We denote such approximate polynomial spectral models as PSM, to distinguish them from the a.i. models themselves. It is important not to think of these PSM as a $\mathcal{O}$ th-order interpolation between a pre-calculated grid of a.i. models (as, e.g., Prugniel et al. 2011 did for a quadratic PSM in three labels), as this would still require $M_{\text {grid }}^{\mathcal{N}} \propto \exp \left(\mathcal{N} \cdot \ln M_{\text {grid }}\right)$ a.i. model calculations. Instead, one should think of determining the (near)-smallest number of a.i. model spectra (specified by $\mathcal{N}$ labels) one needs to calculate in order to construct a $\mathcal{O}$ th-order approximation to the a.i. model spectra. The simplification and speed-up of such a spectral fitting compared to T16 arise from the fact that a single PSM can approximate the a.i. model spectra over a much larger volume in label space. While this shares the idea of a polynomial flux approximation with The Cannon (Ness et al. 2015), it is not data-driven model building.

In the subsequent sections, we first derive that the minimal number of a.i. models needed to construct a PSM of order $\mathcal{O}$ and then illustrate heuristically how well, and over what volumes in label space, these PSMs approximate the a.i. models.

\section{A POLYNOMIAL MODEL APPROXIMATION FOR AB INITIO MODEL SPECTRA OF STARS}

Following T16, we suppose that an a.i. modeling "machinery" can predict the normalized flux of a synthetic spectrum, $f_{a . i .}(\lambda \mid \ell)$, given a set of stellar labels, $\ell$. We assume that the a.i. model spectra change from point to point in label space, but do so smoothly or differentiably at every wavelength. Then the a.i. model spectrum at any $\ell$ sufficiently close to an model grid point $\ell_{*}$ (within a first-order Taylor-sphere or 1OTS, in the nomenclature of T16) can therefore be described with high accuracy by a linear spectral model (LSM; see T16):

$$
f_{\text {lin }}\left(\lambda \mid \ell_{*}+\Delta \ell\right) \simeq f_{a . i .}\left(\lambda \mid \ell_{*}\right)+\Delta \ell^{T} \cdot g\left(\lambda \mid \ell_{*}\right),
$$

where $\boldsymbol{g}\left(\lambda \mid \ell_{*}\right) \equiv \nabla_{\ell} \boldsymbol{f}_{a . i .}\left(\lambda \mid \ell_{*}\right)$.

In principle, specifying an LSM merely requires $1+\mathcal{N}$ model calculations, but T16 showed a factor of a few more is needed to explore the actual extent of the 1OTS. This LSM approximation, $f_{\text {lin }}$, can obviously be generalized to a PSM:

$$
\begin{aligned}
\boldsymbol{f}_{\mathrm{PSM}}\left(\lambda \mid \ell_{*}+\Delta \ell\right) \simeq & \boldsymbol{f}_{a . i .}\left(\lambda \mid \ell_{*}\right)+\Delta \ell^{T} \cdot \boldsymbol{g}\left(\lambda \mid \ell_{*}\right) \\
& +\Delta \ell^{T} \cdot \underline{\underline{\boldsymbol{H}}}\left(\lambda \mid \ell_{*}\right) \cdot \Delta \ell+\ldots,
\end{aligned}
$$

where we will focus on second order, both for astrophysical reasons (it may work well enough) and to avoid cumbersome notation. Such a PSM holds for every one of the $K$ wavelengths $\lambda$. One may think of it as a model with $K$ 0th-order terms, $f_{\mathrm{PSM}}\left(\lambda_{k} \mid \ell_{*}\right)$, then $K \times \mathcal{N}$ first-order terms, and finally $K \times \mathcal{N}(\mathcal{N}+1) / 2$ second-order terms. The number $\mathcal{N}(\mathcal{N}+1) / 2$ arises because of the symmetry of $\underline{\underline{\boldsymbol{H}}}$. In total that makes for

$$
K \times N_{\text {tot }} \equiv K \times(1+\mathcal{N}+\mathcal{N} \cdot(\mathcal{N}+1) / 2)
$$

unknown terms. For more general PSM of order $\mathcal{O}$, one has $K \times N_{\text {tot }}=K \times\left(\begin{array}{c}\mathcal{N}+\mathcal{O} \\ \mathcal{O}\end{array}\right)$.

If we compute a.i. models $f_{a . i .}\left(\lambda \mid \ell_{*}+\Delta \ell\right)$ at $\left(\begin{array}{c}\mathcal{N}+\mathcal{O} \\ \mathcal{O}\end{array}\right)$ different points in label space, $\Delta \ell$, we have created exactly $K \times N_{\text {tot }}$ left-hand side terms to solve exactly for the terms that specify the PSM. Note that, strictly speaking, $\boldsymbol{g}\left(\lambda \mid \ell_{*}\right)$ and $\underline{\underline{\boldsymbol{H}}}\left(\lambda \mid \ell_{*}\right)$ are not exactly the "gradient" and the "Hessian," but merely the first- and second-order coefficients that solve the equation.

Compared to the 1OTS, we have to calculate $1+\mathcal{N} / 2$ times more a.i. models for any one quadratic PSM. However, if the the region in label space around $\ell_{*}$ over which this quadratic PSM works is sufficiently larger, an important speed-up over the (set of) LSM should result. Calculating somewhat more a.i. models than this minimum, and solving Equation (2) in a leastsquares sense, makes for a much better conditioned solution for a PSM, as we show below.

\section{VERIFICATION OF PSM ACCURACY, USING KURUCZ MODELS FOR APOGEE-LIKE SPECTRA}

Strictly verifying the validity of the PSM approximation, like any approximation to a high-dimensional function, would be of enormous computational expense. Here, too, escaping the curse of dimensionality comes at a price: relying on the physically plausible assumption that spectral flux changes can be approximated by polynomials for modest label changes, and settling for heuristic and approximate ways to explore the extent in label space over which a single PSM is useful.

As in T16, we can set out for a pragmatic test of the PSM approximation, using Kurucz model spectra that resemble in resolution and wavelength coverage the APOGEE spectra; the arguments should hold qualitatively for other surveys, but need to be tested case-by-case. In total, the DR12 data release of the APOGEE (Alam et al. 2015; Holtzman et al. 2015) provides 17 labels for each star ( $T_{\text {eff }}, \log g$, and 15 elemental abundances), while fixing $v_{\text {macro }}=6 \mathrm{~km} \mathrm{~s}^{-1}$ and adopting a $\log g-v_{\text {turb }}$ relation for $v_{\text {turb }}$ A quadratic PSM for 19 labels requires a minimum $N_{\text {tot }}=210$ a.i. model calculations. We chose the reference label, $\ell_{*}$, to be the APOGEE DR12 sample median in each of the 19 labels, providing $f_{a . i .}\left(\lambda \mid \ell_{*}\right)$ in Equation (2). The vast majority of targets in APOGEE are disk stars with all $[\mathrm{X} / \mathrm{H}]>-1$, and we restrict our PSM verification to this regime. We then drew $209 \Delta \ell$ at random from the APOGEE DR12 catalog. For the labels $v_{\text {turb }}$ and $v_{\text {macro, }}$, we adopted the same $\log g-v_{\text {turb }}$ relation from APOGEE with a spread of $0.2 \mathrm{~km} \mathrm{~s}^{-1}$ and a distribution in $v_{\text {macro }}$ uniform across $3-8 \mathrm{~km} \mathrm{~s}^{-1}$. We convolved spectra to the APOGEE resolution assuming the combined line-spread function (LSF) from APOGEE and using codes from the APOGEE Python package (Bovy 2016) and continuum-normalized spectra the same way 

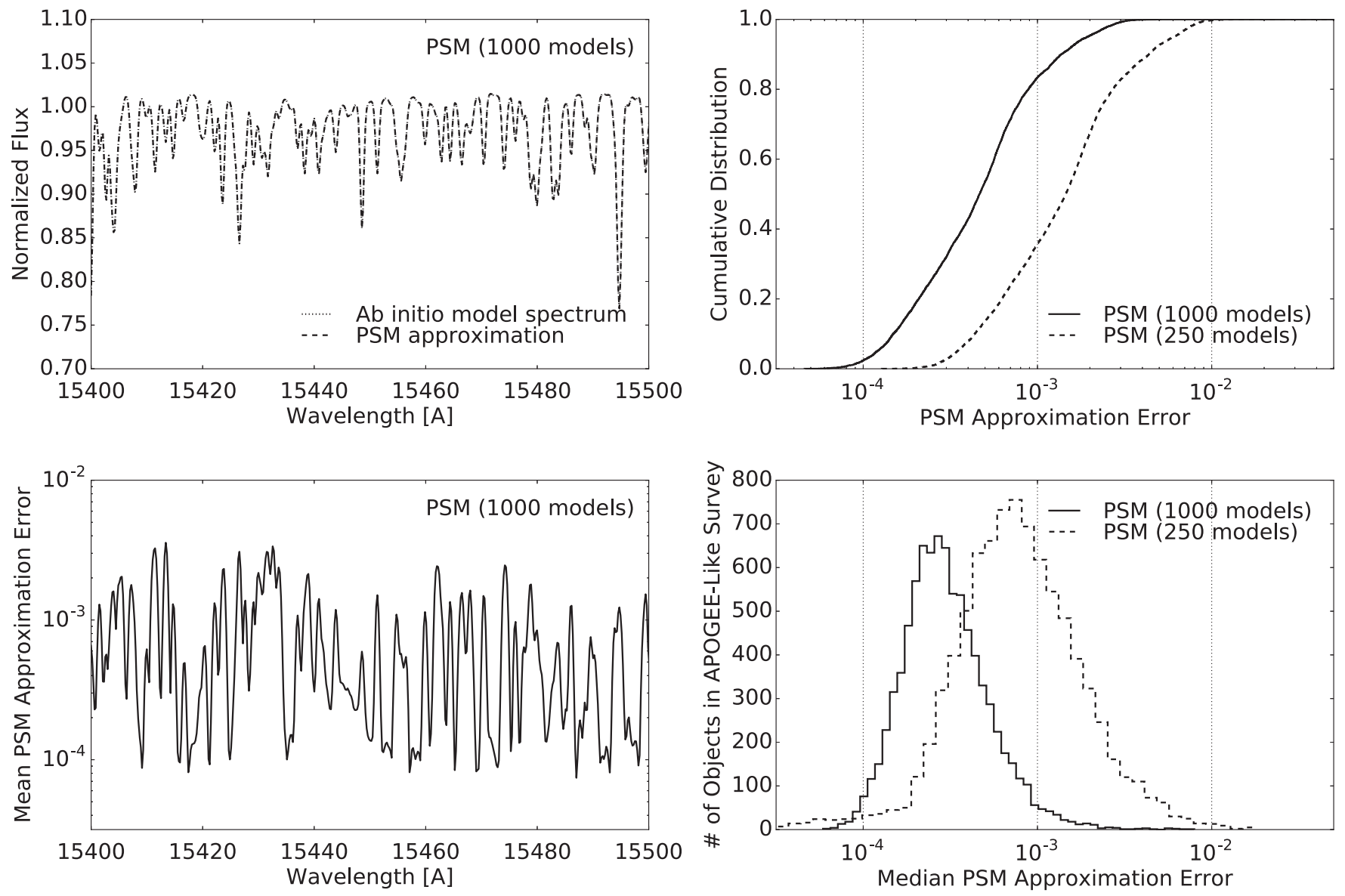

Figure 1. Quality of the (quadratic) PSM approximation: a single PSM was constructed using 250 or 1000 a.i. model spectra (cf. the absolute minimum number of 231), calculated at label points ("objects") drawn randomly from those in the APOGEE survey (Alam et al. 2015; Holtzman et al. 2015). The panels illustrate different PSM-a.i. model comparisons, for 10,000 other objects drawn from the labels of the APOGEE survey. The top left panel shows for a limited wavelength section the average of the exact a.i. model spectra and of the PSM, which appear indistinguishable. The bottom left panel shows the ensemble average (absolute) difference between the a.i. model and the PSM flux (the approximation error), as a function of wavelength. For each one of the 10,000 objects there is a pixel-by-pixel distribution of these approximation errors, which is shown in the top right panel for the pixel-by-pixel average approximation error. The bottom right panel finally shows the distribution across all objects of their (pixel-by-pixel) median approximation error. Note that there are rare cases (objects of very high [Fe/H], where the approximation is only good to a median of $10^{-3}$ ). Taken together, however, this shows that a single PSM approximates the exact $a . i$. model spectra typically to within $10^{-3}$ for objects with a label distribution resembling that of the entire APOGEE survey (which merely serves as an illustration here), over the 10,000 labels of the median. Constructing the PSM from 1000 instead of 250 random label points leads to a better PSM approximation.

as The Cannon (Ness et al. 2015). This provided the remaining 209 left-hand sides of Equation (2) to solve exactly for $\boldsymbol{g}\left(\lambda \mid \ell_{*}\right)$ and $\underline{\underline{H}}\left(\lambda \mid \ell_{*}\right)$, fully specifying $\boldsymbol{f}_{\mathrm{PSM}}\left(\lambda \mid \ell_{*}+\Delta \ell\right)$ from Equation (2).

As expected by construction of the PSM, $f_{\mathrm{PSM}}\left(\lambda \mid \ell_{*}+\Delta \ell\right)$ matches the a.i. model at all the $210 \Delta \ell$ exactly. This minimally constructed PSM also provides good approximations to $f_{a . i .}\left(\lambda \mid \ell_{*}+\Delta \ell\right)$ for other $\Delta \ell$. Empirical experimentation showed that slightly overconstraining Equation (2) worked better: we calculated $f_{a . i .}\left(\lambda \mid \ell_{*}+\Delta \ell\right)$ for 250 and $1000 \Delta \ell$ drawn from APOGEE and solved for the right-hand side of Equation (2) in a least-squares sense to determine the PSM coefficients.

There are two ways in which one can quantify how well the PSM, $f_{\mathrm{PSM}}\left(\lambda \mid \ell_{*}+\Delta \ell\right)$, approximates $f_{a . i .}\left(\lambda \mid \ell_{*}+\Delta \ell\right)$ for any $\Delta \ell$ drawn from APOGEE: how well do the fluxes match, e.g., in a mean absolute deviation? And at what accuracy level does the PSM approximation affect the label recovery?

Figure 1 illustrates how well an a.i. model spectrum of a random star in APOGEE is can be approximated by the PSM in a mean absolute deviation sense. On average, the PSM- predicted flux at any wavelength for a random star within APOGEE is within $10^{-3}$ or $10^{-3.5}$ of that for its a.i. model spectrum, depending on whether we used 250 or 1000 a.i. model calculations to construct the PSM. Figure 2 shows how much (or, how little) the PSM approximation, calculated here on the basis of 250 or 1000 a.i. models, affects the label recovery across an APOGEE-like survey. The labels were recovered by a least-squares fit of the PSM to noiseless a.i. models, fitting all 19 labels simultaneously. These were then compared to the actual labels of the respective a.i. models. With a single PSM, most labels are recovered as accurately as claimed precisions of current spectral surveys. More quantitatively the quality of the PSM label recovery is well tracked by the information content that the spectra contain about any one label: following T16, this is quantified by the Cramer-Rao bound (for $\mathrm{S} / \mathrm{N} \sim 100$ ) using either only the APOGEE wavelength windows for certain elements or the whole spectrum.

The PSM appears heuristically as a better approximation when calculated on the basis of more a.i. model calculations, presumably for two reasons: the system of linear equations in 




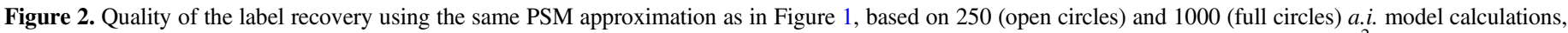

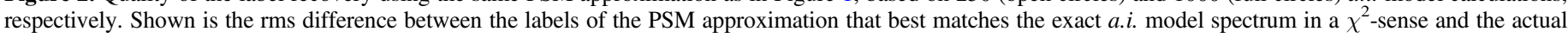

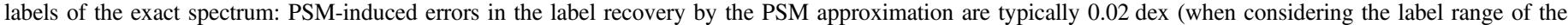





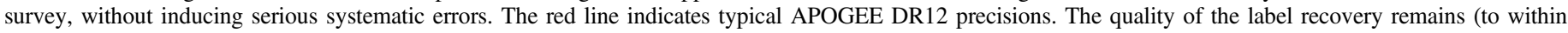
$\sim 10 \%$ of each label's accuracy), even if a number of spectral continuum and line-spread parameters are also fit simultaneously.

Equation (2) becomes better conditioned; and a better sampling of label space better mitigates any breakdown of the polynomial approximation. Both factors must play a role: when we restrict the label range over which we first construct and then test the PSM, the PSM label recovery is even closer to the exact solution. Yet, the PSM constructed on the basis of 1000 (compared to 250) a.i. model calculations is still performing better. How many models to calculate for the PSM construction and over which portion of label space to apply it will therefore depend in practice on the computational expense of the a.i. models and the desired label accuracy. Nonetheless, Figures 1 and 2 demonstrate that with calculating only 250 (or 1000) a.i. models one can construct a single (quadratic) PSM that performs remarkably well in approximating results from exact model spectra at a random 19dimensional label location across much of the APOGEE survey.

\section{PROSPECTS AND LIMITATIONS}

We have shown the advantages for spectral model fitting of generalizing the local linear expansion of a.i. model spectra laid out in T16 to higher order, constructing a PSM that approximates the variations of the predicted spectral flux at each wavelength as a polynomial function of the labels. This reduces the calculation of the model spectra needed in simultaneous fitting of many stellar labels to observed spectra to linear algebra. Compared to established approaches that first calculate grids and then interpolate, the dramatic gain in constructing a PSM comes from the much more benign scaling of the computational effort with increasing label dimension: $\propto\left(\begin{array}{c}\mathcal{N}+\mathcal{O} \\ \mathcal{O}\end{array}\right)$, or $\propto \mathcal{N}^{2}$ for a quadratic model with $\mathcal{O}=2$, as opposed to $\propto \exp (\mathcal{N} \cdot \ln M)$. The way these PSM are constructed are mathematically very much analogous to the data-driven The Cannon (Ness et al. 2015), where a quadratic spectral model is derived form observed spectra.
The arguments here provide a systematic guidance for the size of the required training set in The Cannon: we should expect the training set size to scale as (as a multiple) $\left(\begin{array}{c}\mathcal{N}+2 \\ 2\end{array}\right)$, or $\propto \mathcal{N}^{2}$; this makes it plausible that The Cannon could constrain 19 labels from a training set of 10,000 (Casey et al. 2016).

The heuristic verification of the PSM approximation, along with the framework laid out in T16, means that there should be no longer serious technical obstacles to determining stellar labels in large surveys to what amounts to fitting all labels with a.i. model spectra simultaneously. The accommodation of label correlation facilitates the extraction of abundance information from blended spectral features. We find from the gradient spectra that $80 \%$ of the spectrum's information on a label is typically spread over $30 \%$ of all pixels and is not just in narrow spectral windows (T16). For any given data set this should allow higher precision and accuracy. PSM also allows us to treat parameters of the experimental setup, such as the continuum fit or the spectral quasi-LSF, as stellar labels and to fit them simultaneously.

Of course, constructing PSMs is not a panacea; while a single PSM appears to suffice for the much of APOGEE survey, this is presumably because APOGEE has targeted stars in a rather restricted portion of label space: giant stars in a narrow temperature range. Yet, even there, constructing a separate PSM for the metal-poor regime may be advisable, as small model flux differences cause larger label recovery errors. Second, it is probably worth exploring the PSM approach to higher order in at least some of the labels. Perhaps most importantly, any fitting based on a.i. spectral models can only work as well as the physics behind them. Insufficient atomic data or the restrictions of the LTE approximation remain untouched by the ideas laid out here. Nonetheless, we feel that T16 and this Letter lay out a path that may help in doing justice 
to the enormous information content of present and future stellar spectroscopy surveys.

Y.S.T. acknowledges support from NASA grant NNX15AR83H and is grateful to the MPIA and the DFG through the SFB 881 (A3) for their hospitality and financial support. C.C. acknowledges support from NASA grant NNX13AI46G, NSF grant AST-1313280, and the Packard Foundation. H.W.R.'s research contribution is supported by the ERC Grant Agreement No. [321035]. The computations in this Letter were partially run on the Odyssey cluster supported by the FAS Division of Science, Research Computing Group at Harvard University. The computational work also used the Extreme Science and Engineering Discovery Environment (XSEDE), which is supported by National Science Foundation grant number ACI-1053575. We thank Bob Kurucz for developing and maintaining programs and databases without which this work would not be possible.

\section{REFERENCES}

Alam, S., Albareti, F. D., Allende Prieto, C., et al. 2015, ApJS, 219, 12

Allende Prieto, C., Beers, T. C., Wilhelm, R., et al. 2006, ApJ, 636, 804

Allende Prieto, C., Fernández-Alvar, E., Schlesinger, K. J., et al. 2014, A\&A, 568, A7

Bovy, J. 2016, ApJ, 817, 49

Casey, A. R., Hogg, D. W., Ness, M., et al. 2016, ApJ, submitted (arXiv:1603.03040)

Frebel, A., \& Norris, J. E. 2015, ARA\&A, 53, 631

Freeman, K., \& Bland-Hawthorn, J. 2002, ARA\&A, 40, 487

García Pérez, A. E., Allende Prieto, C., Holtzman, J. A., et al. 2016, AJ, 151,144

Holtzman, J. A., Shetrone, M., Johnson, J. A., et al. 2015, AJ, 150, 148

Ness, M., Hogg, D. W., Rix, H.-W., Ho, A. Y. Q., \& Zasowski, G. 2015, ApJ, 808,16

Prugniel, P., Vauglin, I., \& Koleva, M. 2011, A\&A, 531, A165

Recio-Blanco, A., Bijaoui, A., \& de Laverny, P. 2006, MNRAS, 370, 141

Rix, H.-W., \& Bovy, J. 2013, A\&ARv, 21, 61

Sheinis, A., Anguiano, B., Asplund, M., et al. 2015, JATIS, 1, 035002

Smiljanic, R., Korn, A. J., Bergemann, M., et al. 2014, A\&A, 570, A122

Ting, Y.-S., Conroy, C., \& Rix, H.-W. 2016, ApJ, in press (arXiv:1602.06947) 